\title{
Observation of Damage Growth in Compressively Loaded Laminates
}

\author{
The phenomenological aspects of composite-panel-failure \\ under compressive in-plane loading and low-velocity \\ transverse impact are studied experimentally via real-time \\ recording of the failure-propagation event
}

\section{by H. Chai, W.G. Knauss and C.D. Babcock}

ABSTRACT-An experimental program to determine the phenomenological aspects of composite-panel failure under simultaneous compressive n-plane loading and low-velocity transverse impact $[0-75 \mathrm{~m} / \mathrm{s}(0-250 \mathrm{ft} / \mathrm{s})]$ is described. Highspeed photography coupled with the shadow-moiré technique is usec to record the phenomenon of failure propagatior. The information gained from these records, supplemented by plate sectioning ard observatior, for interior damage, has provided information regarding the failure-propagation mechanism.

The results show that the failure process can be divided roughly into two phases. In the first phase the plate is inpacted, and the resulting response causes interlaminar separation. In the second phase the local damage spreads to the uncamaged portion of the plate through a combination of laminae blickling and further delamination.

\section{List of Symbols}

$$
\begin{aligned}
n & =\text { fringe number } \\
V & =\text { impact velocity } \\
w & =\text { out-of-plane displacement } \\
w / n & =\text { fringe constant } \\
\Delta t & =\text { time interval between frames } \\
\epsilon_{0} & =\text { compression strain ('load') }
\end{aligned}
$$

\section{Introduction}

Graphite/epoxy laminates are a special class of composites which enjoy a definite strength-to-weight advantage over many standard engineering materials used in aerospace applications. This advantage is somewhat lessened, however, by their sensitivity to operational hazards such as low-velocity impact by foreign objects. Impact may cause delamination damage since the strength of the plate in its thickness direction is quite low. Under compressive loadings this local delamination may grow and lead to a global failure of the structure at a load well below the design level. Structures may be subjected to both highcompression load and impact damage, possibly simultaneously. This situation is exemplified, for instance, by

H. Chai, formerly Graduate Student and subsequently Research Fellow at California Institute of Technology, is Visiting Scientist, AFWAL/MLBM, Wright-Patterson AFB, Dayton, OH 45433. W.G. Knauss (SESA Member) and C.D. Babcock (SESA Member) are Professors of Aeronautics, California Institute of Technology, Pasadena, CA 91125.

Original manuscript submitted: July 13, 1982. Final version received: May 25, 1983. a stone, kjcked up on a runway, impacting a lower-wing skin during landing or take-off. The wing motion generated by the landing induces compression load cycles in the skin so that the local damage induced by impact can propagate to the undamaged portions of the skin.

The effect of impact damage on the strength degradation in compressively loaded laminates can be characterized by a threshold for catastrophic failure such as that shown by curve A or B in Fig. 1. This curve is generated by impacting panels preloaded in compression and assessing the post-impact results. Panels that fail completely upon impact generate data for strains ('loads') and impact velocities above the threshold curve whereas those which did not fail generate data points below this curve. Also shown in Fig. 1 is a graph of the damage area

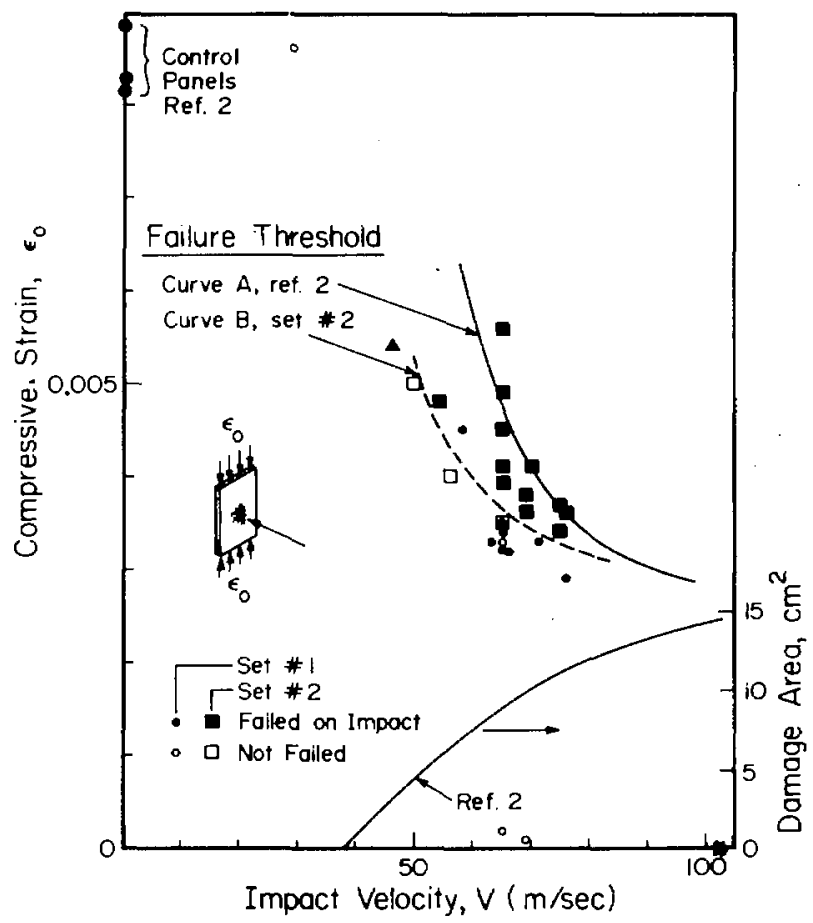

Fig. 1-Impact conditions for T300/5208 laminates. Impactor is $12.7-\mathrm{mm}$ aluminum sphere. Triangular symbol represents panel (set No. 2) which failed due to global plate response 
caused upon impact vs. impact velocity. This curve was generated by impacting unloaded panels and determining the extent of damage using the $\mathrm{C}$-scan technique. Figure 1 shows that for small impact velocities [i.e., $V<40 \mathrm{~m} / \mathrm{s}$ $(130 \mathrm{ft} / \mathrm{s})]$ there is no damage and no apparent strength reduction in the laminate. For larger impact velocities, the damage area increases while the plate strength decreases. At impact velocity on the order of $100 \mathrm{~m} / \mathrm{s}(330 \mathrm{ft} / \mathrm{s})$ the plate strength approaches a minimum which is only about 30 percent of the undamaged plate strength. It should be noted that variations in test parameters such as plate thickness, layup and projectile mass can lead to variations in the nominal value of projectile speed at which strength degradation begins to take place or strength degradation approaches a maximum. ${ }^{1}$

Several investigations on this topic have been performed also by other researchers. ${ }^{1-5}$ In all these works only postmortem examination of the impacted plates was considered. While the effect of impact damage was fairly well established in Refs. 1-5, little information was revealed concerning the process of damage spreading. The large amount of energy released during catastrophic failure usually leads to a considerable disintegration of the test specimen making it difficult if not impossible to deduce the failure process from such examinations. The mechanism of delamination growth in unloaded glass/ epoxy laminates subjected to impact was studied in Ref. 6 using high-speed photography. Unlike in Ref. 6 where the growth of the delamination is attributed to the stress waves generated by impact, here we are primarily con- cerned with the spread of delamination under the action of the compressive load.

In the present work, which is condensed from parts of Refs. 8 and 9, we determine the damage-growth mechanism via real-time recording of the impact event. The material, a T300/5208 graphite/epoxy laminate, is typical of the configuration proposed for future heavily loaded primary structures and has stiffness properties similar to those of wing skins in existing transport aircrafts. The model projectile is a $12.7-\mathrm{mm}(0.5$-in.) diam aluminum sphere propelled normal to the plane of the plate at a velocity in the range of $0-75 \mathrm{~m} / \mathrm{s}(0-250 \mathrm{ft} / \mathrm{s})$, its mass and speeds simulating momenta typical of low-velocity impact hazards that can occur in service. High-speed photography is used to record the propagating failure from several positions. In some tests back surface (unimpacted surface) and front surface, or back surface and edge surface of the plate are taken simultaneously using two high-speed cameras. The records of the back and front surface are made in conjunction with the shadow-moire technique to obtain a full-field, out-of-plane deformation history of the impacted plates.

\section{Test Apparatus}

A photograph of the test setup is shown in Fig. 2 and includes the panel support fixture, the loading machine, the impact device (air-gun), the light sources and two high-speed cameras. A review of the main components follows.

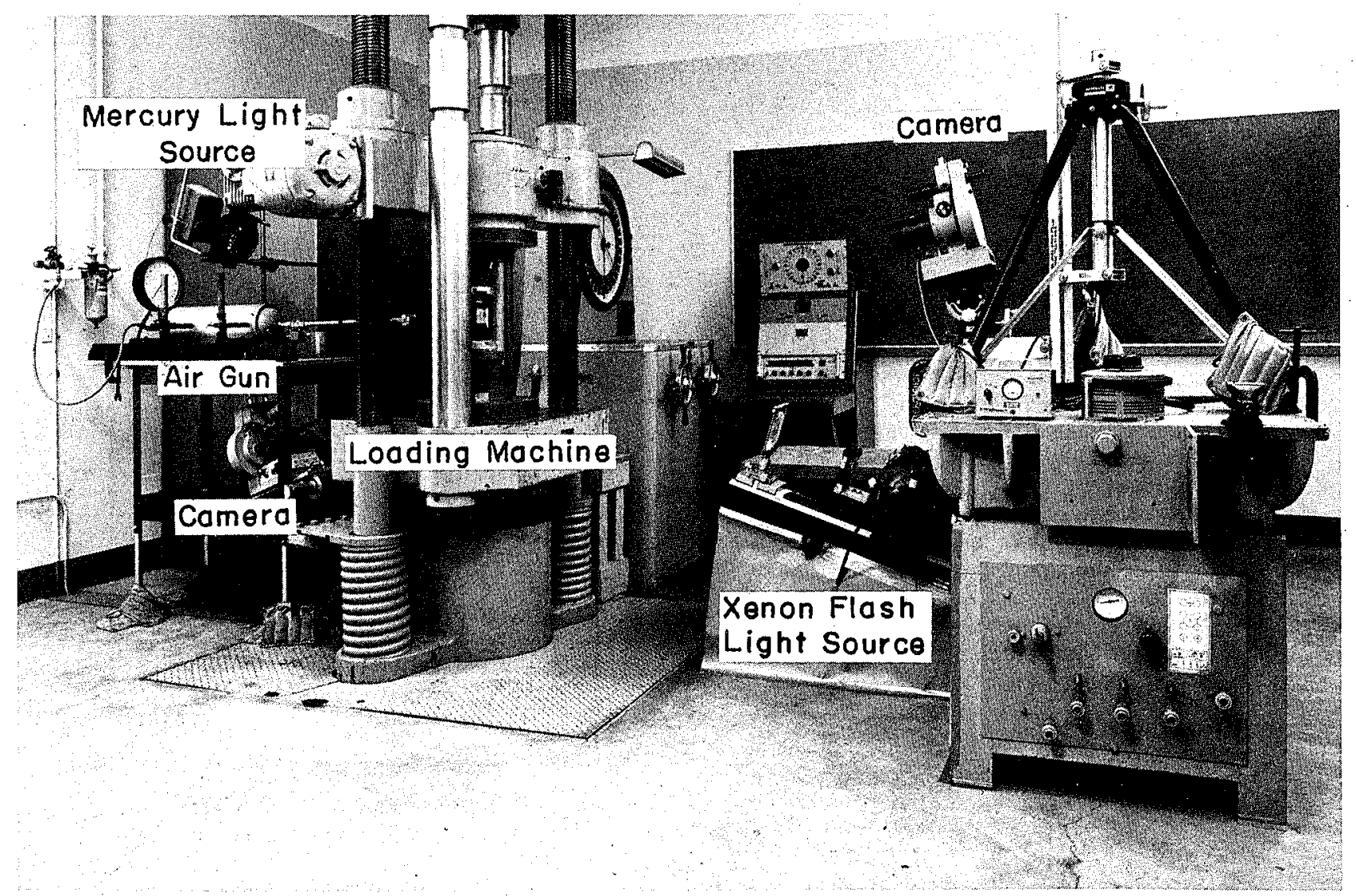

Fig. 2-Impact and recording test setup 

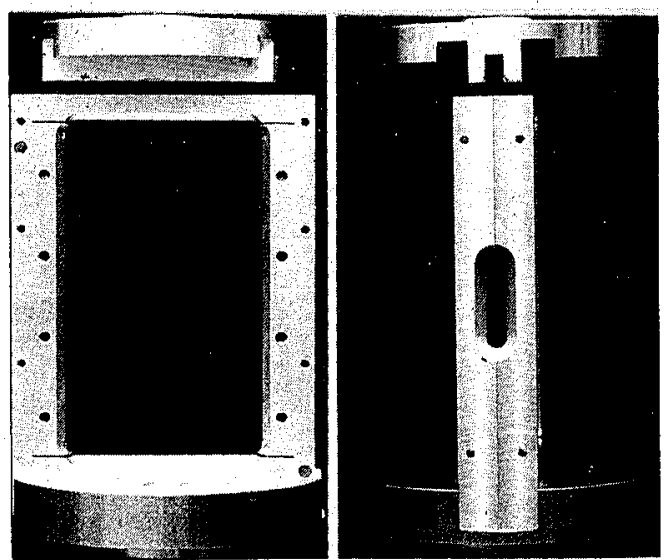

Fig. 3-Test-panel support fixture

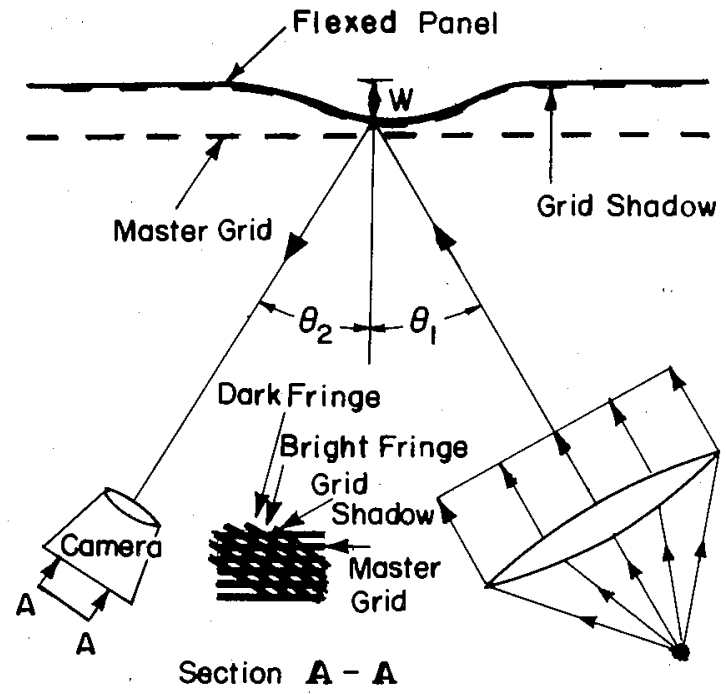

Fig. 4-Shadow-moiré optical setup

\section{Panel Fixture}

Two fixtures are used to support two sets of graphite/ epoxy panels having different sizes, i.e., $20.3 \times 10.2 \times$ $0.60 \mathrm{~cm}(8 \times 4 \times 0.24$ in. $)$ in set No. 1 , and $25.4 \times$ $15.2 \times 0.60 \mathrm{~cm}(10 \times 6 \times 0.24$ in. $)$ in set No. 2. The 'small' fixture is shown in Fig. 3. The upper and lower edges of the panel which transmit the load are bonded to aluminum plates in grooves filled with a bonding agent (Devcon A). The depth of the grooves in the 'small' and 'large' panel fixtures are $1 \mathrm{~cm}(0.4$ in.) and $2.5 \mathrm{~cm}$ (1.0 in.), respectively. Both sides of the specimens are held by narrow strips. These strips are adjustable along the length of the specimen to ensure a close, but nonbinding, support.

\section{Loading Machine}

The load is applied to the specimen by a hydraulic testing machine capable of exerting loads up to 150 metric tons. A spherical base support transmits the load to the specimen to assure uniformity in the load distribution. The latter, measured by as many as eight strain gages, is within \pm 3 percent of the mean.

\section{Impact Device}

The projectile is accelerated by a simple air-gun consisting of a compressed-air reservoir, solenoid activated valve and a $38 \mathrm{~cm}$ (15 in.) long barrel. The projectile velocity is controlled by adjusting the pressure in the reservoir and is measured by twice interrupting a light beam monitored by a photodiode. The time interval between beam interruptions is measured by a counter. With this device, the scatter in the impact velocity is less than \pm 1 percent.

\section{Shadow Moiré}

Moiré is an optical phenomenon based on the interaction of light formed with two gratings; one grating is stationary and undeformed (the master grating) while the other deforms with the specimen. As the specimen grating
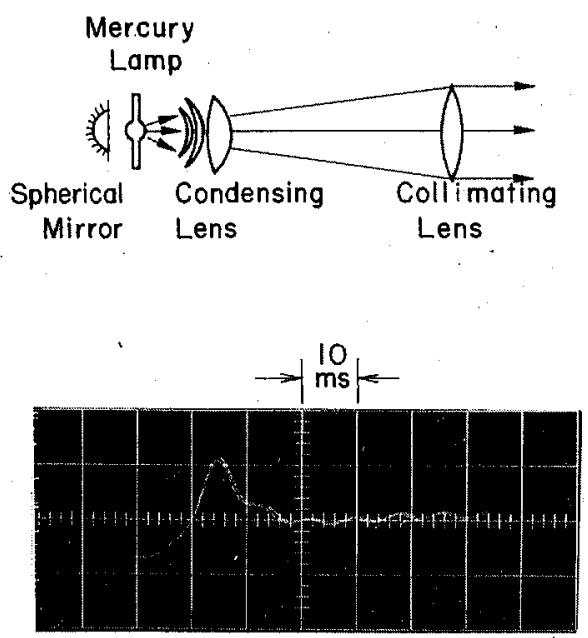

(b) Pulsed Mercury Lamp
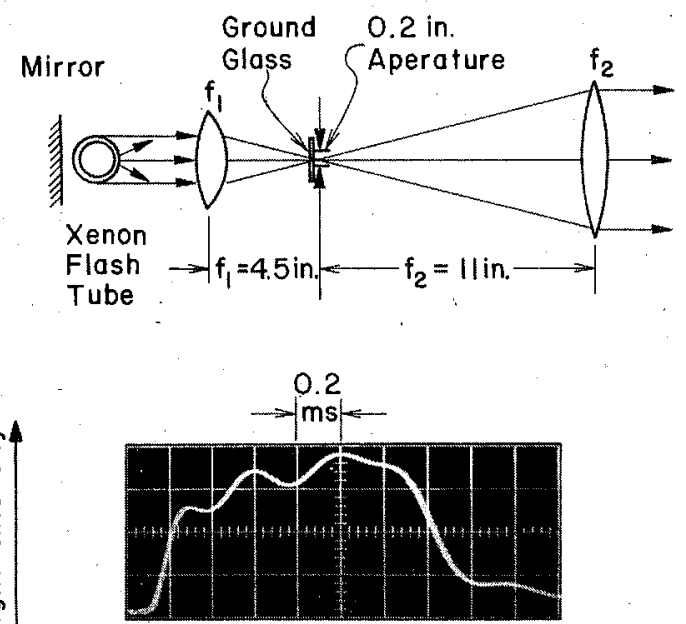

Fig. 5-Optical arrangement (upper parts) and light-intensity history (lower parts) of two light sources 
deforms, a fringe pattern related to the surface deformation of the specimen is observed. In the shadow moire, a grid placed close to the specimen surface serves as the master grating while its shadow on the matte-reflective surface of the specimen, formed by an incident collimated light beam, serves as the specimen grating. The normal displacement $w$ is given by (for details, see for example Ref. 7)

$$
\frac{w}{n}=\frac{p}{\tan \theta_{1}+\tan \theta_{2}}=\text { fringe constant }
$$

where $n$ is the fringe number, $p$ is the grating pitch and $\theta_{1}$ and $\theta_{2}$ are the angles formed between the normal to the plate and the directions of the incident and viewing light, as shown in Fig. 4.

A Ronchi-ruled grid with $4 \mathrm{l} / \mathrm{mm}(100 \mathrm{l} / \mathrm{in}$.) is used as the master grating. The grid is placed close to the specimen surface [2-4 mm (0.08-0.16 in.)] for sufficient fringe contrast. Because the grating is destroyed in each test, replicas of the grating are formed by contact printing the master onto high-contrast, high-resolution Kodak Ortho Plate PFO. The matte reflective surface for the shadow moire is achieved by painting the plate surface with Krylon 'silver' spray paint.

\section{Light Source}

A Xenon flash tube, powered by a bank of capacitors which is capable of discharging 4000 joules is used in recording the impact event. The optical setup shown in Fig. 5(a) is used in order to provide a collimated and uniform light beam at the specimen surface. The flash history, controlled by adjusting the discharging rate of the capacitors, is selected to produce a pulse as shown in the record in Fig. 5(a). The flash tube is triggered by the projectile using a simple wire-breaking circuit. A 200-w Mercury bulb is used in conjunction with the Xenon flash tube on occasions where multiple but simultaneous recordings are made. The bulb is pulsed to $800 \mathrm{w}$ during the impact to increase its light intensity, according to the record shown in Fig. 5(b).

\section{High-speed Camera}

One, and on occasions two, 16-mm Hycam (Red Lake Laboratory, Model K2054BE) high-speed framing cameras were used to recórd the impact event. In one camera a quarter-frame assembly has been installed to

\section{TABLE 1-TEST CONDITIONS 1}

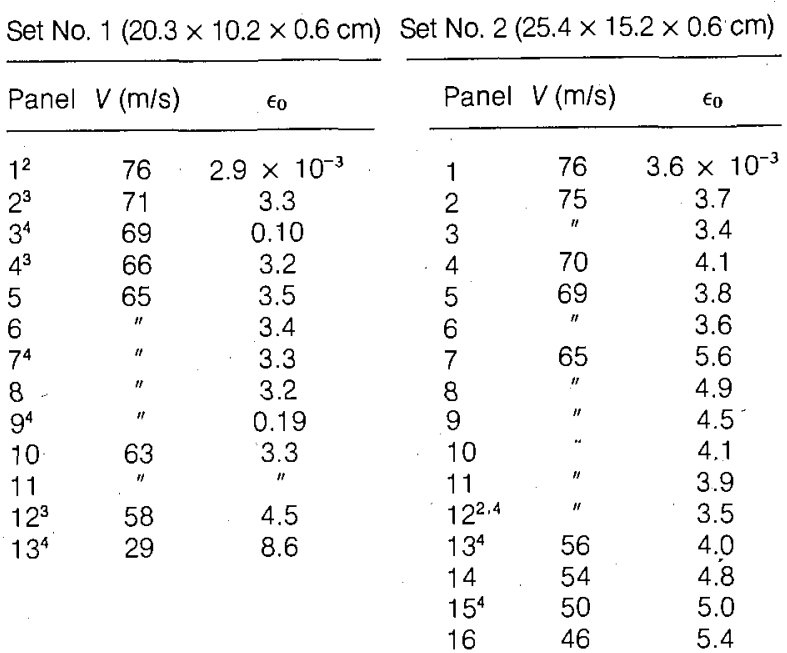

\footnotetext{
${ }^{1}$ Except where otherwise mentioned, back-surface moiré is employed, and the panels failed completely upon impact.

${ }^{2}$ Back surface and edge of panel are recorded simultaneously.

${ }^{3}$ Back and front surfaces of panel are recorded simultaneously.

${ }^{4}$ Panel did not fail upon impact.
}

Fig. 6(a) and (b)Impact damage in a 6-mm thick graphitel epoxy laminate: (a) C-scan of an unloaded plate impacted at $76 \mathrm{~m} / \mathrm{s}$, (b) through-thethickness section along $90 \mathrm{deg}$ of the panel in (a)

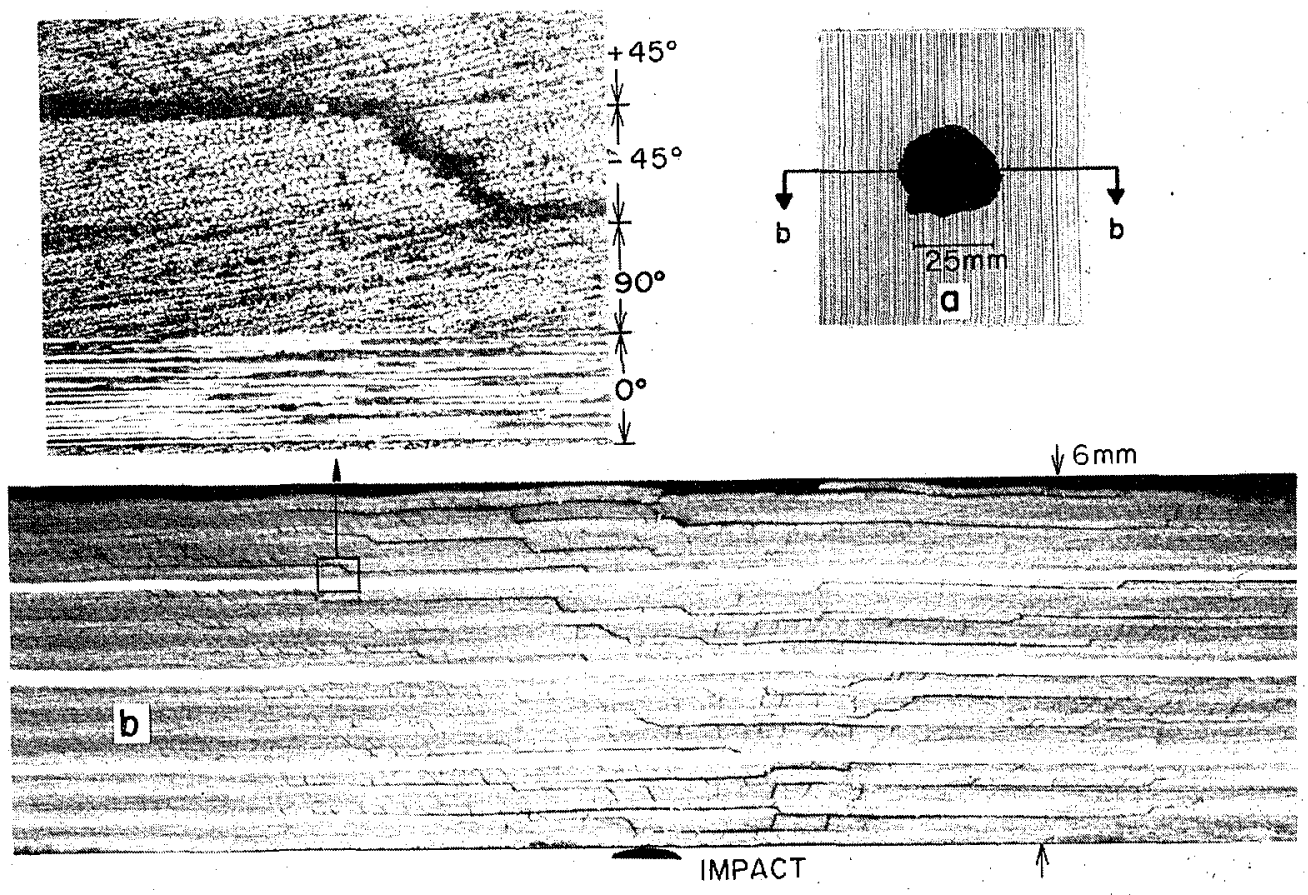


increase its recording rate up to 40,000 frames/s. The exposure time, controlled by an interchangeable shutter, is selected in the range of 2.5-5 $\mu \mathrm{s}$. In cases where two cameras are used simultaneously, a common timing light pulse displayed on both films synchronizes the two records (Kodak pan film, 2484 high-speed film, rated at 800 A.S.A.).

\section{Test Procedure and Test Data}

The panels tested in this investigation were furnished through NASA-Langley.* The specimens were fabricated from commercially available tapes made from unidirectional Thornel-300 graphite fibers pre-impregnated with Narmco-5208 epoxy resin (28-percent resin content). The tapes were laid up to form a 48-ply $(+45 /-45 / 0 / 0 /+45 /$ $-45 / 0 / 0 /+45 /-45 / 0 / 90)_{2 s}$ laminate.**

The panels, supported by the fixtures, are impacted at the center while under compressive load applied along the 0-deg ply direction. The impact event is photographed. Table 1 summarizes the test conditions and the photographic procedure. The test results are displayed in Fig. 1 along with a rough estimate of a curve indicating the failure threshold of panels belonging to set No. 2 (curve B). The lower segment of this curve is drawn relatively accurately. The high-speed photographs of the two 'large' panels closest to this segment (i.e., panel No. 3 and No. 6 in Table 1) exhibit a phase of initial delay in the damage growth. This is in contrast to the rest of the panels in that segment of the curve which show damage growth without such a delay, the growth rate which increases with increasing strain. Therefore, the test conditions for the two panels under discussion represent nearly threshold data. The rest of the curve could not be drawn with such an accuracy. Note first that establishing

*Courtesy of J.H. Starnes, Jr

**The nominal ply properties are: longitudinal modulus $=131.0 \mathrm{GN} / \mathrm{m}^{2}$ $\left(19.0 \times 10^{6} \mathrm{PSI}\right)$, transverse modulus $=13.0 \mathrm{GN} / \mathrm{m}^{2}\left(1.89 \times 10^{6} \mathrm{PSI}\right)$, shear modulus $=6.4 \mathrm{GN} / \mathrm{m}^{2}\left(0.93 \times 10^{6} \mathrm{PSI}\right)$, major Poisson's ratio $=$ 0.38. The calculated composite stiffnesses can be found in Refs. 8 and 9. a failure threshold curve was not our prime motivation in this work. Secondly, we recognize that the limited supply of test material is inadequate to define such a curve well. The available data, however, are sufficient to indicate the existence of limit behavior in the threshold curve at both relatively low and relatively high projectile speeds; at these velocity ranges the failure strain seems to be little affected by changes in the impact speed. It is also clear that in between these two extremes a transition region exists in the threshold curve where the failure strain degrades rapidly with increasing impact velocity. Also shown in Fig. 1 is a failure threshold curve obtained in Ref. 2 (curve A) for the same material as in this work but for a slightly different plate size $[25 \times 13 \times 0.68 \mathrm{~cm}$ $(10 \times 5 \times 0.27$ in. $)]$. The differences observed between the data of Ref. 2 (curve A) and our data (curve $B$ and the data for the panels of set No. 1) are probably due to lot variations in material properties.

\section{Interior-damage Characterization}

Some panels that did not fail catastrophically upon impact were examined ultrasonically and/or sectioned through the plate thickness at different locations and examined microscopically for interior damage. Figure 6(a) shows an ultrasound C-scan* record of a plate impacted while under zero load. From this record, the damage area appears roughly circular in shape with approximately $3 \mathrm{~cm}$ (1.2 in.) in diameter. A section of this panel along the 90-deg ply direction and through the impact point is shown in Fig. 6(b). The damage in Fig. 6(b) is characterized by delamination and intraply cracking extending across the full thickness of the plate. This damage is extended laterally more toward the unimpacted panel face than toward the impacted face.

A section through the impact point and along the 0-deg ply direction of a plate which was loaded during impact

*The authors are grateful to J.H. Starnes, Jr. of NASA-Langley for providing the C-scans.

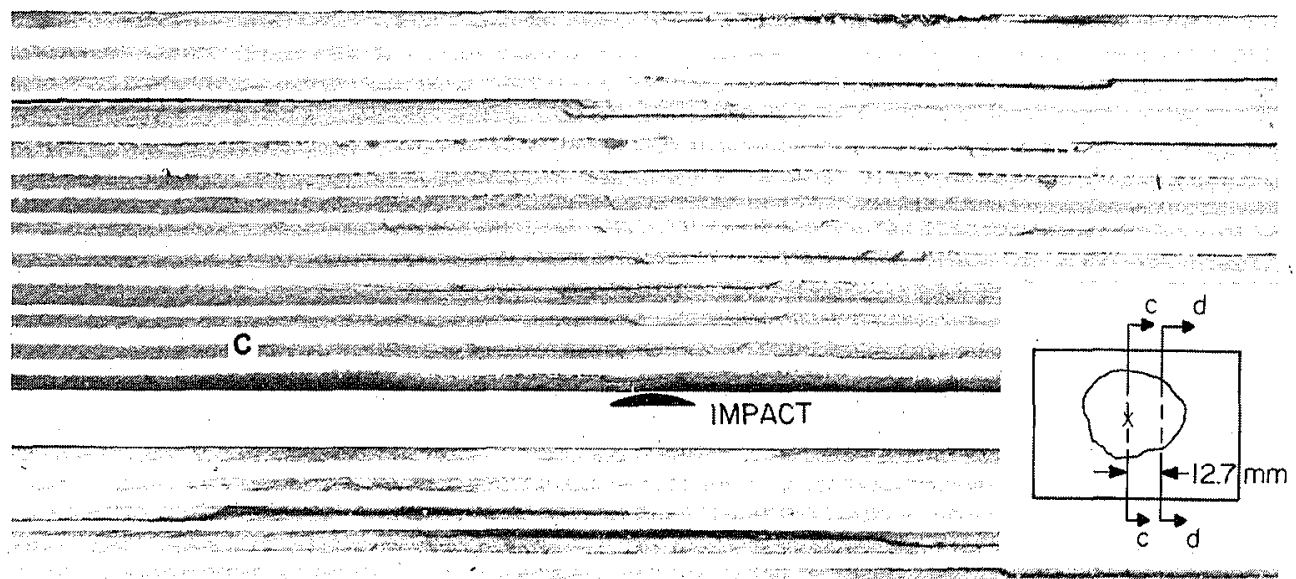

Fig. 6(c) and (d)Two sections along 0 deg, 12.7-mm apart, of a panel loaded to $\epsilon_{0}=0.0035$ and impacted at $65 \mathrm{~m} / \mathrm{s}$ 
but did not fail catastrophically. is shown in Fig. 6(c). The appearance and distribution of the damage in this section is quite similar to that of the unloaded case shown in Fig. 6(b), though the damage extends over a larger area, probably due to the effect of the load. A section parallel to this section, but $12.7 \mathrm{~mm}(0.5 \mathrm{in}$.) away, is shown in Fig. 6(d). This view indicates that the delaminated area is confined only to the vicinity of the surface opposite to the impact face. Since the conditions at impact were close to the threshold condition, the initial damage area is similar to that of the panels that did fail completely upon impact.

\section{High-speed Photography Results and Discussion}

We turn now to a consideration of the real-time surface deformation of the impacted plates. A moiré pattern recorded from the back-surface showing the failure process of a panel belonging to set No. 1 is given in Fig. 7(a). The test conditions for this panel $[V=71 \mathrm{~m} / \mathrm{s}$ $\left.(233 \mathrm{ft} / \mathrm{s}), \epsilon_{0}=0.0033\right]$ are at the far right of the threshold curve where the strength degradation due to impact is most pronounced, and are typical of the majority of the tests for this set of panels. The load is only about 20 percent of the buckling load for this panel. This selection reflects our desire to study the local growth process as independent as possible from the global plate response due to impact, the latter increases with increasing applied load. The displacements in Fig. 7(a) are summarized in Fig. 8 as a function of horizontal and vertical (loadparallel) coordinates which originate at the impact point. These displacements include both permanent and vibra-
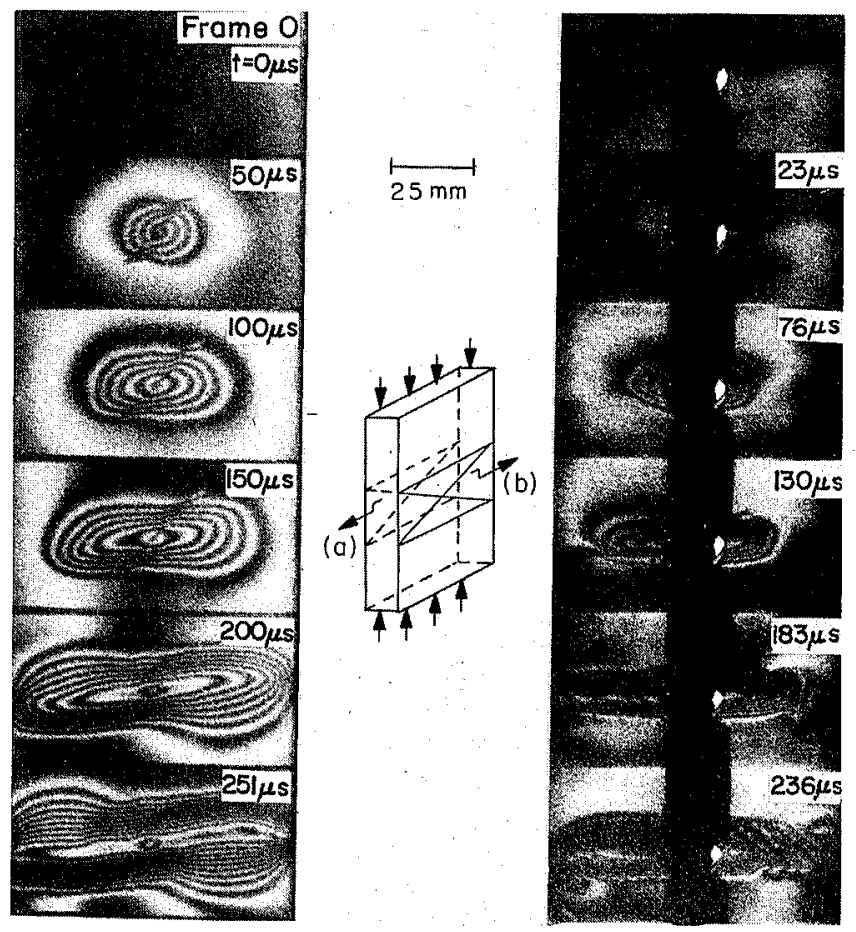

Fig. 7-Simultaneous back- and front-surface-moire motion pictures showing panel (set No. 1) failure, $\epsilon_{0}=0.0033$, $V=71 \mathrm{~m} / \mathrm{s}$; (a) back-surface, $w / n=0.32 \mathrm{~mm}$, (b) frontsurface, $w / n=0.31 \mathrm{~mm}$ tional deformations. However, an analysis* and tests" have shown that the contribution of the vibration term to the total displacement is small so that the moire fringes can be viewed as representing permanent deformation or damage.

Frame 0 in Fig. 7(a) represents the condition just prior to impact and has been assigned the time $t=0$. In the subsequent frame the impact caused a bulging of the surface near the impact region with a peak displacement on the order of $1 \mathrm{~mm}(0.05 \mathrm{in}$.). Based on the results of the interior-damage characterization, this bulging of the surface is interpreted as a local buckling of the delaminated area under the action of the compressive load.** Subsequent frames, at $50 \mu \mathrm{s}$ time interval, show that this buckled region propagates laterally (normal to the loading direction) at a velocity on the order of $300 \mathrm{~m} / \mathrm{s}(1000 \mathrm{ft} / \mathrm{s})$ with the peak displacement increasing during the growth.

While the growth history of the bulge at the panel back surface is fairly clear from Fig. 7(a), no direct information is revealed concerning the damage extension in the interior of the plate during the growth process. It appears appropriate, therefore, to record simultaneously the two opposite surfaces of the panel during the impact event. Figure 7(b) shows the front surface moire version of the panel in Fig. 7(a). The grid center is perforated by a 15$\mathrm{mm}$ (0.6-in.) diam hole to allow for impact without destroying the grid. The dark area in this series of photographs is the shadow of the gun barrel. The time relationship between the two motion pictures, evaluated with the aid of the common timing light pulse, is marked in Fig. 7(b). Impact has occurred in the second frame in Fig. 7(b) $(t=23 \mu \mathrm{s})$. Subsequent frames show that a characteristic deformation*** has developed and propagated across the panel with little growth in the 'vertical' direction. The existence of deformation on both faces of the panel at a given time indicates that the propagating damage extends through the plate thickness.

To further examine the relation of the surface deformation to internal damage, a $4-\mathrm{cm}$ (1.5-in.) long clearance was cut along the edge of the panel fixture as shown in Fig. 3. A simultaneous recording of the edge and the back surface of a specimen is shown in Fig. 9. Note that the characteristic deformation in the upper sequence has reached the left edge of the panel at approximately $300 \mu \mathrm{s}$ after impact. This event occurs at about the same time as the appearance of the first crack at the edge, i.e., the frame at $345 \mu \mathrm{s}$ in the lower sequence in Fig. 9. The results of this experiment suggest that the internal-damage front practically coincides with the first or lowest order fringe in the moiré pattern. This association will be used later in this work in the construction of the delamination growth rate in Fig. 11. Subsequent frames in both motion pictures show only the final stages in the collapse of the panel.

The test data for the panels of set No. 2 cover a relatively broad range in the transition region in the threshold curve (Fig. 1) which allows for evaluation of the

\footnotetext{
*The analysis, based upon orthotropic-plate theory and a Hertzian contact model for the impact-load history, has indicated that the maximum displacement expected under conditions that simulate the test conditions would be at most $0.3 \mathrm{~mm}(0.01 \mathrm{in}$.) or approximately a single fringe in Fig. $7(a)$.

**It is noted that impact tests on unloaded panels reported in Ref. 9 have shown that no such bulging of the surface under the impact site has occurred.

***Based on post-mortem examination of the deformed panel, and also on an estimate of the projectile contact duration, this deformation is interpreted to be in the direction of the bulge at the back-surface.
} 
more detailed aspects of the damage-growth phenomenon. Figure 10 shows a series of four back-surface motion pictures for four panels impacted at the same velocity but loaded to different degrees. The failure process for these tests is much the same as for the panels of set No. 1 but the relatively large dimension of these panels and the
Fig. 8-Out-of-plane displacement corresponding to Fig. 7(a)
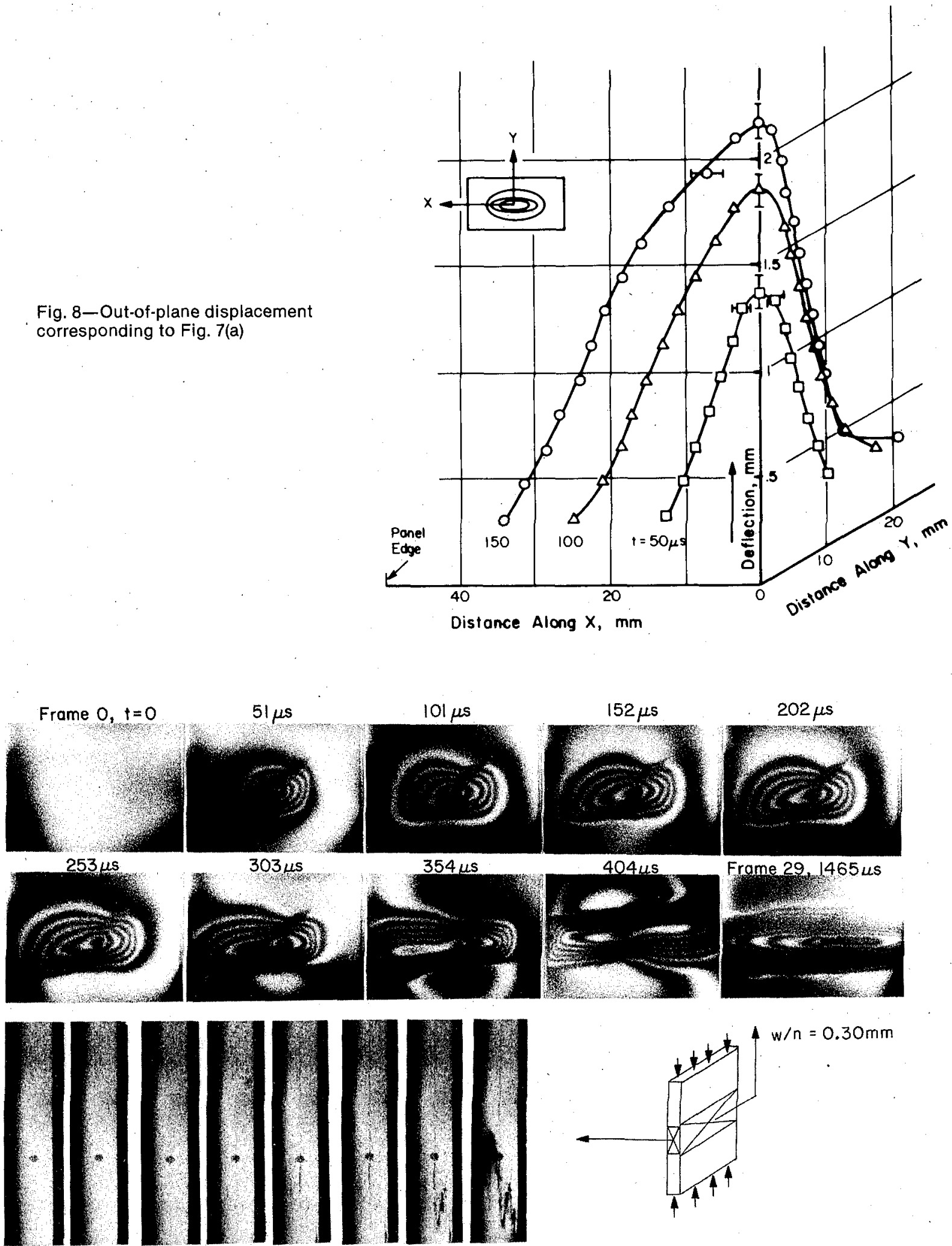

$t=14 \mu \mathrm{s}$

$289 \mu \mathrm{s}$

$1612 \mu \mathrm{s} 1667 \mu \mathrm{s}$

Fig. 9-Simultaneous back-surface (upper sequence) and edge motion pictures (panel of set No. 1), $\epsilon_{0}=0.0029$, $\mathrm{V}=76 \mathrm{~m} / \mathrm{s}$ 


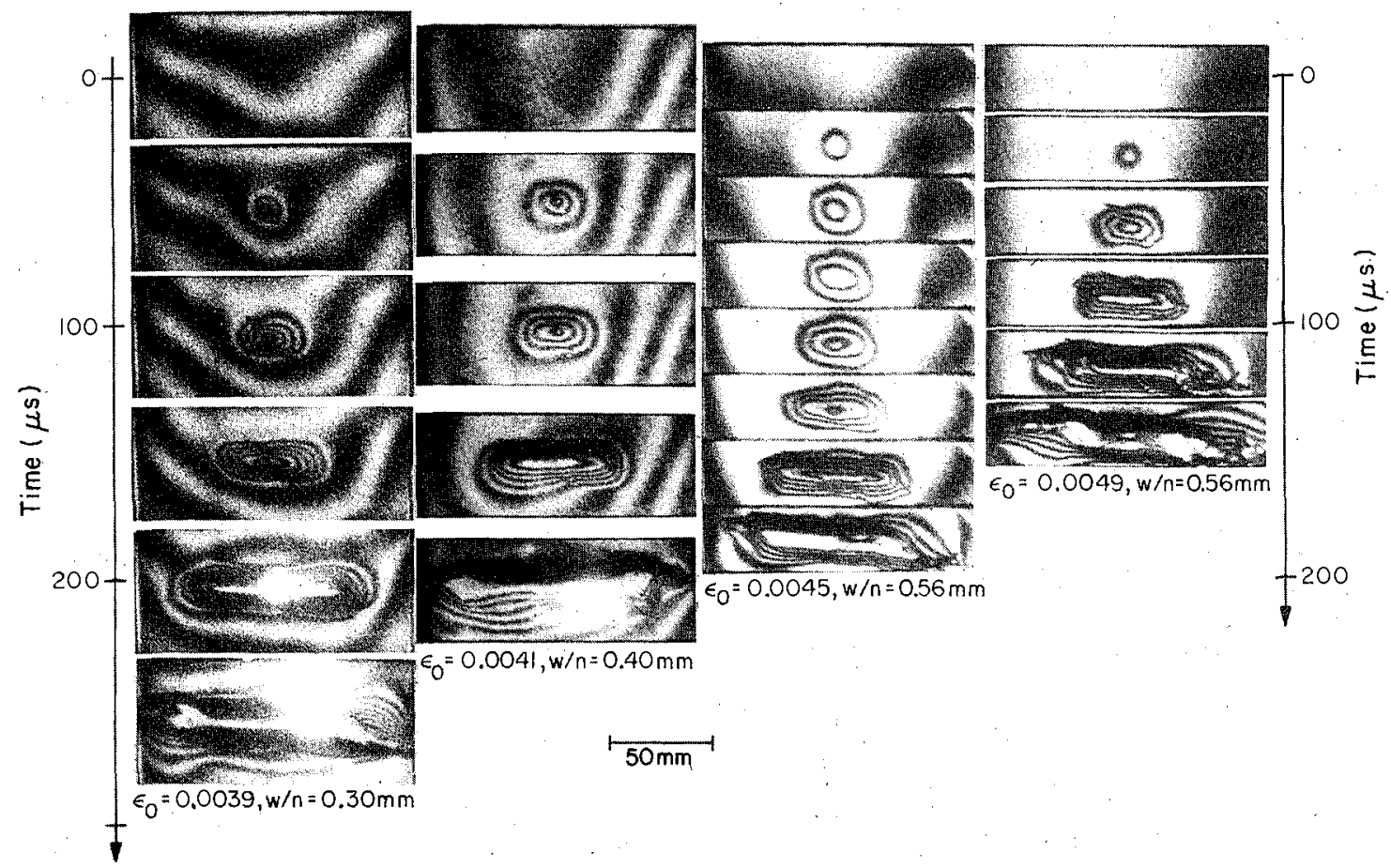

Fig. 10-Damage propagation under different loadıngs in panels (set No. 2) impacted at $\mathrm{V}=65 \mathrm{~m} / \mathrm{s}$

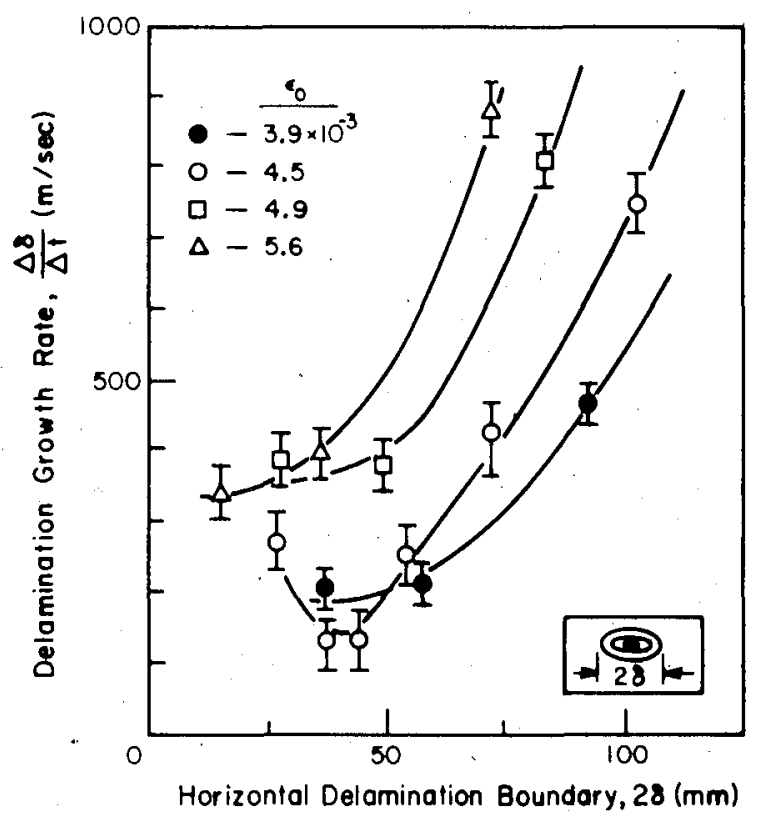

Fig. 11-Delamination growth rate for panels (set No. 2) loaded to different degrees but impacted at $\mathrm{V}=65 \mathrm{~m} / \mathrm{s}$

range in the loading allow the evaluation of the dynamics of the growth process. Figure 10 shows that the rate of damage growth increases as the damage spreads, and is larger for higher compression strains. Figure 11 gives the growth-rate history of the delamination area for four different panels. The growth rate is evaluated based on the amount of the 'horizontal' extension of the damaged area, as defined in the insert in Fig. 11, over the time
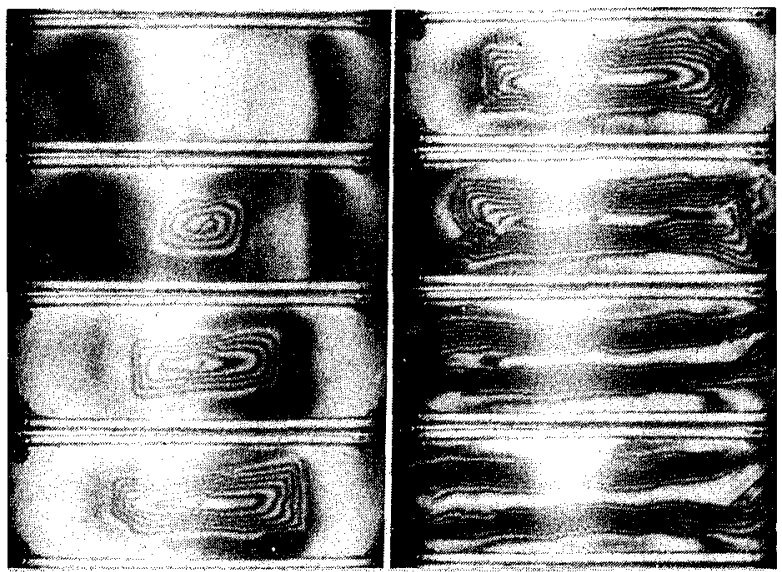

Fig. 12-A 'rectangular'-type delamination boundary (pane of set No. 2), $w / n=0.42 \mathrm{~mm}, \Delta t=55.6 \mu \mathrm{s}, \epsilon_{0}=0.0036$, $\mathrm{V}=76 \mathrm{~m} / \mathrm{s}$

interval between two adjacent frames in the moiré sequence. All tests exhibit a phase of relatively slow growth followed by a 'smooth' and rapid growth as the delamination area spreads. A systematic transition between these two phases of growth is not strictly apparent from the data presented, but it is seen that the phase of rapid propagation starts after the damage has extended over about $5 \mathrm{~cm}(2 \mathrm{in}$.). For a delamination of twice this size, the growth rate is more than doubled, reaching a value of up to $850 \mathrm{~m} / \mathrm{s}(2800 \mathrm{ft} / \mathrm{s})$ for the test with the highest load.

Some panels have failed in a manner which differs from that shown before. Figure 12 shows a 'rectangular'-type boundary of a propagating delamination which differs from the smooth elliptical ones observed in other 

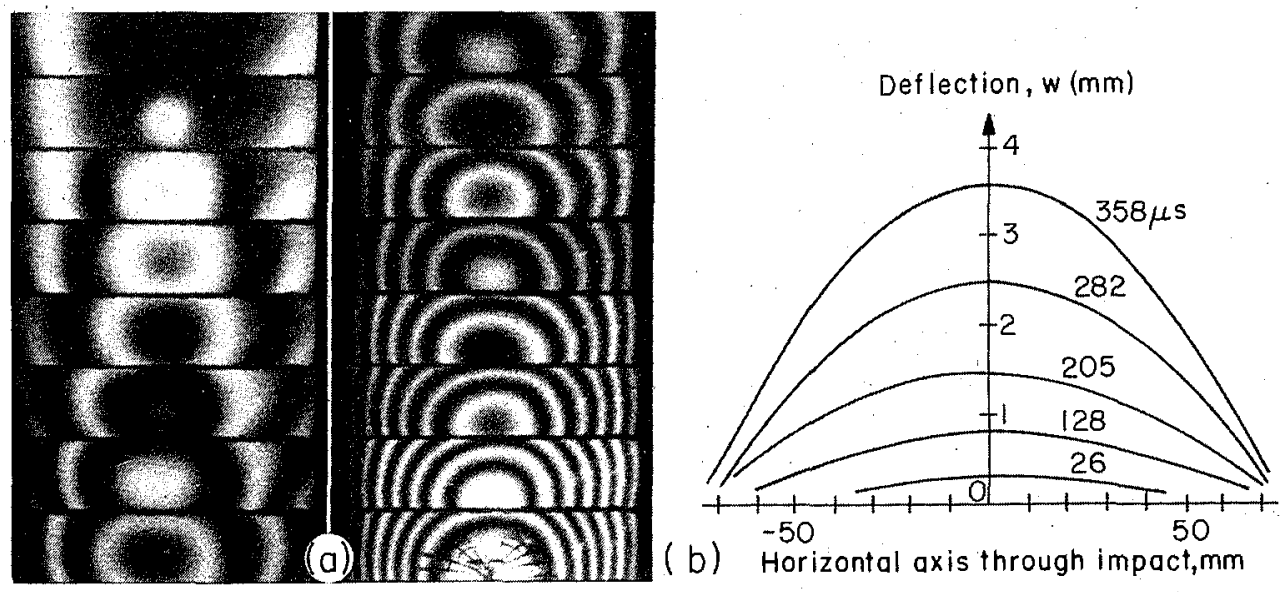

Fig. 13-Failure of a panel (set No. 2) due to global plate response: (a) back. surface moiré showing the initial phase in the development of failure (complete failure occurred later in time), $w / n=0.56$ $\mathrm{mm}, \Delta t=25.6 \mu \mathrm{s}$, $\epsilon_{0}=0.0054, V=46$ $\mathrm{m} / \mathrm{s}$; (b) out-of-plane deflection under the impact point vs. time of the panel in (a)

experiments of similar test conditions. Most likely, it is associated with a delamination at a different interface. We note, however, that this change in the growth geometry has not altered the growth characteristics (i.e., as before the growth is predominantly normal to the loading and the out-of-plane deflection increases with increasing delamination size).

Another uncommon test result is shown in Fig. 13. The test conditions for this panel, represented by the triangular symbol in Fig. 1, correspond to a relatively small impact velocity and a relatively large load $\left(\epsilon_{0}=0.0054\right)$. The relatively large time scale of the failure [Fig. 13(a)] (note that this figure shows only the initial phase in the development of failure) and the sizable deflections [Fig. 13(b)] indicate that this panel has not failed as a result of localdamage spread but in a dynamically induced globalbuckling mode. The result of this test indicates that the failure of a panel depends on the buckling stiffness, or, equivalently, on the ratio of applied load to laminate buckling load. This assessment is manifested by the test result of panel No. 13 of set No. 1 (see Table 1 and Fig. 1). Although loaded to a higher level than the larger panel of Fig. 13 (i.e., $\epsilon_{0}=0.0086$ ), it did not fail globally upon impact. It is noted that the ratio of applied load to buckling load for this panel is approximately $0.55^{*}$ which is smaller than the corresponding ratio for the panel of Fig. 13, of approximately 0.75 .

\section{Conclusions}

We have presented a qualitative and quantitative examination of damage propagation in compressively loaded composite laminates subjected to low-velocity impact. Such impact can produce delamination. Under in-plane compression the delamination may buckle and propagate unstablely through a combination of delamination and buckling. This spreading, which occurs normal to the loading direction, starts at a relatively low speed and accelerates into a 'smooth' and rapid delamination growth. The propagation speed of the delamination region is influenced by the strain level and speeds up to $850 \mathrm{~m} / \mathrm{s}$

*Utilizing available buckling curves for orthotropic laminates, ${ }^{10}$ and assuming that the loaded edges of the panels are clamped while the unloaded edges are simply supported, the buckling strains for the 'large' and 'small' panels are 0.007 and 0.016 , respectively.
$(2800 \mathrm{ft} / \mathrm{s})$ are recorded for the largest strain applied $\left(\epsilon_{0}=0.0056\right)$.

The consistency in the growth behavior during the rapid-growth phase observed in repeated tests, indicates that this phase can be separated in time from the damagegeneration and initial-growth phase as well as from the global-plate response due to impact. This finding is useful in formulating a model of damage growth. Apart from failure by local-damage spreading, a panel may also fail by global-buckling mode. The existence of this mode of failure depends on the global-buckling load of the laminate which, in the present work, is controlled by the laminate dimension.

\section{Acknowledgments}

This work was supported by the National Aeronautics and Space Administration under Grant NSG-1483. The authors are appreciative of this support and wish to acknowledge the encouragement and interest of James $H$. Starnes, Jr., NASA Langley, in pursuing this work.

\section{References}

1. Byers, B.A., Behavior of Damaged Graphite/Epoxy Laminates Under Compression Loading," NASA CR 159293, (Aug. 1980).

2. Rhodes, M.D., Williams, J.G. and Starnes, J.H., Jr., "LowVelocity Impact Damage in Graphite-Fiber Reinforced Epoxy Laminates," 34th Annual Conf. Reinforced Plastics/Composite Institute, Soc. of the Plastic Industry, Inc., New Orleans, LA (Jan. 29-Feb. 2, 1979).

3. Starnes, J.H., Jr., Rhodes, M.D. and Williams, J.G., "The Effect of Impact Damage and Circular Holes on the Compressive Strength of a Graphite-Epoxy Laminate," NASA TM 78796.

4. Rhodes, M.D., "Damage Tolerance Research on Composite Compression Panels," Selected NASA Res. in Composite Matls. and Structures, NASA CP 2142, 107-142 (Aug. 1980).

5. Williams, J.G., Anderson, M.S., Rhodes, M.D., Starnes, J.H., Jr. and Stroud, W.J., "Recent Developments in the Design, Testing and Impact-Damage Tolerance of Stiffened Composite Panels," NASA TM 80077 (April 1979).

6. Takeda, N., Sierakowski, R.L., Ross, C.A. and Malvern, L.E., "Delamination-crack Propagation of Ballistically Impacted Glass/Epoxy Composite Laminates," EXPERIMENTAL MECHANICS, 22 (1), 19-25 (Jan. 1982).

7. Theocaris, S.T., "Moiré Fringes in Strain Analysis," Pergamon Press (1969).

8. Chai, H., "The Growth of Impact Damage in Compressively Loaded Laminates," PhD Thesis, Cal. Institute of Tech. (March 1982).

9. Knauss, W.G., Babcock, C.D. and Chai, H., "Visualization of Impact Damage of Composite Plates by Means of the Moiré Technique," NASA CR 159261 (April 1980).

10. Dale, F.A. and Smith, R.C.T., "Grid Sandwich Panels in Compression," Australian Council Aeronaut: Rept., ACA-16, Melbourne (April 1945). 